\title{
Next-Generation Sequencing Gene Panels and "Solo" Clinical Exome Sequencing Applied in Structurally Abnormal Fetuses
}

\author{
Montse Pauta ${ }^{a}$ Berta Campos $^{\mathrm{b}}$ Maria Segura-Puimedon ${ }^{\mathrm{b}}$ Gemma Arcac \\ Alfons Nadald $^{d}$ Albert Tubau $^{\text {e Silvia Pina Perez }}{ }^{f}$ Edda Marimong \\ Lourdes Martín $^{\text {h }}$ Eva López-Quesada' Joan Sabriàg Begoña Muñoz ${ }^{j}$ \\ Esperanza Garciak Fernanda Paz Y. Miño' Virginia Borobiol Olga Gomez' \\ Elisenda Eixarch ${ }^{\text {l, } m}$ Monica Lopez ${ }^{\mathrm{h}}$ Montserrat Comas Rovira ${ }^{\mathrm{f}}$ Antoni Borrella,

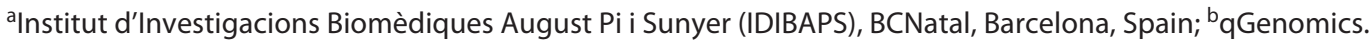 \\ Esplugues de Llobregat, Catalonia, Spain; 'DepartmentofNeonatology, BCNatal, Hospital Clinic Barcelona,

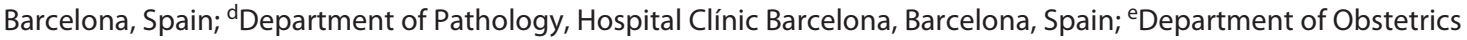 \\ and Gynecology, Son Llàtzer Hospital, Palma de Mallorca, Spain; fDepartment of Obstetrics and Gynecology, \\ Parc Taulí Hospital, Sabadell, Catalonia, Spain; ${ }^{9}$ Department of Obstetrics and Gynecology, Hospital Sant Joan \\ de Déu, BCNatal, Esplugues de Llobregat, Catalonia, Spain; 'Department of Obstetrics and Gynecology, Hospital \\ Joan XXIII de Tarragona, Catalonia, Spain; 'Department of Obstetrics and Gynecology, Hospital Mútua de Terrassa, \\ Terrassa, Spain; 'Department of Obstetrics and Gynecology, Hospital Sant Joan de Reus, Reus, Spain; ${ }^{k}$ Department \\ of Obstetrics and Gynecology, Hospital Consorci Sanitari de Terrassa, Terrassa, Spain; 'Department of Obstetrics \\ and Gynecology, BCNatal, Hospital Clínic de Barcelona, Barcelona, Spain; ${ }^{\mathrm{m} C e n t r e ~ f o r ~ B i o m e d i c a l ~ R e s e a r c h ~ o n ~ R a r e ~}$ \\ Diseases (CIBER-ER), Madrid, Spain
}

\section{Keywords}

Congenital malformations - Fetal genetic analysis - Genetic disorder - Prenatal diagnosis - Single gene disorders .

Exome sequencing

\begin{abstract}
Objective: The aim of the study was to assess the diagnostic yield of 2 different next-generation sequencing (NGS) approaches: gene panel and "solo" clinical exome sequencing (solo-CES), in fetuses with structural anomalies and normal chromosomal microarray analysis (CMA), in the absence of a known familial mutation. Methodology: Gene panels encompassing from 2 to 140 genes, were applied mainly in
\end{abstract}

karger@karger.com www.karger.com/fdt

Karger $\stackrel{\text { ' }}{5}$

GOPEN ACCESS
C 2021 The Author(s).

Published by S. Karger AG, Basel

This is an Open Access article licensed under the Creative Commons Attribution-NonCommercial-4.0 International License (CC BY-NC) (http://www.karger.com/Services/OpenAccessLicense), applicable to the online version of the article only. Usage and distribution for commercial purposes requires written permission. persistent nuchal fold/fetal hydrops and in large hyperechogenic kidneys. Solo-CES, which entails sequencing the fetus alone and only interpreting the Online Mendelian Inheritance in Man genes, was performed in multisystem or recurrent structural anomalies. Results: During the study period (2015-2020), 153 NGS studies were performed in 148 structurally abnormal fetuses with a normal CMA. The overall diagnostic yield accounted for $35 \%(53 / 153)$ of samples and $36 \%(53 / 148)$ of the fetuses. Diagnostic yield with the gene panels was $31 \%(15 / 49)$, similar to $37 \%(38 / 104)$ in soIo-CES. Conclusions: A monogenic disease was established as the underlying cause in $35 \%$ of selected fetal structural anomalies by gene panels and solo-CES.

(C) 2021 The Author(s).

Published by S. Karger AG, Basel
Correspondence to:

Antoni Borrell, aborrell@ clinic.cat 


\section{Introduction}

Structural fetal anomalies occur in approximately $2.5 \%$ of pregnancies [1] and most of them can be identified by ultrasound examination during pregnancy. When a fetal structural anomaly is detected by ultrasound, normally a chromosomal microarray analysis (CMA) is offered after amniocentesis, chorionic villi sampling, or fetal blood sampling because it provides up to a $6-10 \%$ incremental diagnostic yield $[2,3]$ above the $14 \%$ yield of a conventional karyotype [4].

The advent of high-throughput next-generation sequencing (NGS) allows sequencing the whole human genome in a single assay, while classic Sanger sequencing was limited to 1,000 nucleotides. To explore the frequency of monogenic disorders associated with fetal structural anomalies, whole-exome sequencing (WES) has been preferred over whole-genome sequencing because human exons account for only $2 \%$ of the whole genome and can explain $85 \%$ of monogenic disorders, and due to the cost and complexity of whole-genome sequencing analysis. The first prenatal diagnosis using WES was achieved in 2013 by the Baylor College group, when a mutation in the NIPBL gene associated with Cornelia de Lange syndrome was identified in a malformed fetus [5]. Applied to structurally abnormal fetuses with a normal CMA, a larger WES series of unselected fetal structural anomalies gave a $10-11 \%$ diagnostic yield $[6$, 7], lower than those observed in small series, including cases selected after genetic review that ranged from 10 to $32 \%[8,9]$.

In 2015, our group initially applied NGS technology to diagnose monogenic disorders, using a gene panel if a clinically available phenotype-driven panel was available. Since 2016, when the observed fetal phenotype did not overlap with a previously described phenotype, we applied a simplified exome sequencing approach called "solo" clinical exome sequencing (solo-CES). The aim of the present study was to report our experience with the use of NGS gene panels and solo-CES in fetuses with selected structural anomalies and normal CMA.

\section{Material and Methods}

This is a prospective study conducted from January 2015 to October 2020 in which NGS studies were offered to pregnant women and partners presenting with selected structurally abnormal fetuses, normal CMA and no familial molecular diagnosis. This cohort includes consecutive cases.

NGS Applied in Structurally Abnormal Fetuses

\section{Fetal Phenotyping}

Our center is a tertiary referral center with an average of 400 cases of fetal abnormalities per year. Ultrasound examinations were performed by expert Fetal Medicine specialists in the participating centers, while extended echocardiogram, neurosonography, or magnetic resonance imaging contributed to the diagnostic approach when appropriate. Pregnant women with any fetal anomaly were offered an invasive procedure and quantitative fluorescent polymerase chain reaction (QF-PCR) and CMA was performed on fetal samples. In terminated or stillborn fetuses, postmortem studies were also recommended. In continuing pregnancies, postnatal follow-up was assessed.

\section{DNA Extraction and NGS}

Fetal DNA was extracted from chorionic villi, amniotic fluid, or fetal blood, although in some samples, cell culturing was required as a preliminary step. Remaining fetal DNA from CMA was sent to the NGS laboratory together with peripheral blood of both biological parents, with the exception of pregnancies conceived by gamete donation. Genomic DNA was obtained from fetal samples and peripheral blood lymphocytes of both parents to be used for subsequent segregation studies, if required. DNA quality was determined by measuring optical density with a DeNovix DS-11. Human exome was enriched using the MedExomeSeqCap EZ assay (Roche-Nimblegen, Pleasanton, CA, USA) and massively sequenced using NextSeq 500 (Illumina, San Diego, CA, USA) equipment. An average $30 \times$ coverage depth was achieved along the target regions (exons and \pm 75 intronic nucleotides flanking the exon-intron boundaries of all genes).

\section{Gene Panel Analysis}

If a clinically available phenotype-driven panel was available, a gene panel was used. "Gene panel" was defined as the NGS test that analyzes multiple genes at once, ranging from 2 to 140 in our study, associated with a specific fetal phenotype. Gene panels offer a middle ground between sequencing just a single gene, and sequencing every gene in the genome. Six gene panels were applied in more than one case: (a) RASopathies-hydrops panel in unexplained fetal hydrops/persistent nuchal fold, defined as an increased nuchal translucency persisting in the second trimester as an increased nuchal fold ( $>5 \mathrm{~mm}$ at $15-17$ weeks and $>6 \mathrm{~mm}$ at $18-22$ weeks)/ cystic hygroma; (b) Congenital Anomalies of the Kidney and Urinary Track (CAKUT) panel [10] in large hyperechogenic kidneys; (c) Osteogenesis imperfecta panel in suspicion of osteogenesis imperfecta (OI); (d) Tuberous sclerosis panel in single or multiple cardiac rhabdomyomas; (e) Craniosynostosis panel in fetal craniosynostosis; and (f) Lissencephaly panel in fetal lissencephaly. Furthermore, 5 gene panels were used only once: Cornelia de Lange panel, visceral myopathy panel, Cardiomyopathy panel, Fanconi panel [11], and CHARGE panel. The number of genes studied in each of the 11 gene panels are shown in Table 1, and the genes and the name of the laboratory are listed in online supplementary Table 1 (see www.karger.com/doi/10.1159/000519701 for all online suppl. material). In skeletal dysplasias other than OI, exome sequencing was preferred over a gene panel analysis.

\section{Solo-Clinical Exome Sequencing}

During the period 2016-2020, when the observed fetal phenotype did not overlap with a previously described phenotype, we applied a simplified exome sequencing approach called solo-CES, given that 
Table 1. Diagnostic yield achieved by NGS gene panels and solo-CES

\begin{tabular}{llll}
\hline NGS gene panel (31\%: 15/49) & & & \\
\hline Gene panel type & $\begin{array}{l}\text { Included } \\
\text { genes }(n)\end{array}$ & $\begin{array}{l}\text { Definitive diagnoses } \\
(N / N)\end{array}$ & $\begin{array}{l}\text { Diagnostic yield } \\
\text { (\%) }\end{array}$ \\
\hline RASopathies-hydrops panel & 130 & $8 / 23$ & 35 \\
CAKUT panel & 140 & $4 / 7$ & 57 \\
Osteogenesis imperfecta panel & 22 & $1 / 4$ & 25 \\
Tuberous sclerosis panel & 2 & $1 / 4$ & 25 \\
Craniosynostosis panel* & 11 & $1 / 4$ & 25 \\
Lissencephaly panel** & 24 & $0 / 2$ & 0 \\
Cornelia de Lange panel & 5 & $0 / 1$ & 0 \\
Visceral myopathy panel & 12 & $0 / 1$ & 0 \\
Cardiomyopathy lanelt & 119 & $0 / 1$ & 0 \\
Fanconi panel & 22 & $0 / 1$ & 0 \\
CHARGE panel & 2 & $0 / 1$ & 0 \\
\hline Solo-CES (37\%: 38/104) & & & \\
\hline Indication & & Definitive diagnoses & Diagnostic yield \\
& & $(N / N)$ & $(\%)$ \\
\hline Multisystem anomalies & & $28 / 69$ & 41 \\
Recurrent anomalies & & $3 / 12$ & 25 \\
Skeletal dysplasia & & $5 / 10$ & 50 \\
Complex CNS anomalies & & $2 / 8$ & 25 \\
Increased nuchal translucency & & $0 / 4$ & 0 \\
\hline Complex cardiac defects & & & \\
\hline
\end{tabular}

CAKUT, congenital anomalies of the kidney and urinary tract; CNS, central nervous system; NGS, next-generation sequencing; solo-CES, solo-clinical exome sequencing. * In one of the 3 fetuses with a negative panel result, soloCES was subsequently performed as complex CNS anomaly. ${ }^{* *}$ Both fetuses had a negative panel result and underwent a subsequent solo-CES, 1 as a complex CNS anomaly and the second as a recurrent anomaly. ${ }^{\dagger}$ This fetus had a negative panel result and solo-CES was subsequently performed as a complex CNS anomaly. ${ }^{\S} \mathrm{After}$ a negative panel result, solo-CES was subsequently performed as a multisystem anomaly.

the fetus alone was sequenced and only the Clinical Genomics Database and Online Mendelian Inheritance in Man (OMIM) genes were interpreted [12]. Solo-CES was offered in consecutive cases presenting with: (a) Multisystem anomalies (at least 2 major anomalies from different anatomical systems); (b) recurrent anomalies (a similar major anomaly in consecutive pregnancies); and (c) severe skeletal dysplasias with at least bilateral upper and/or lower limbs micromelia below-3 SD, not resembling OI. Solo-CES was occasionally performed in 3 further anomaly types: (a) complex central nervous system anomalies after fetal brain specialists suspected a monogenic defect; (b) complex cardiac defects after fetal heart specialists suspected a monogenic defect; and (c) increased nuchal translucency above the 99th percentile as a self-funded personal choice.

During the final 26 months of the study (July 2018-October 2020), pregnancies affected with multisystem or recurrent structural fetal anomalies from 7 further participating centers were also recruited and studied by means of solo-CES. Informed consent was obtained independently from each parent, including an option of opting out of being informed of secondary and incidental findings. At the pretest genetic counseling session, prospective parents were informed about the proposed genetic test and advantages and limitations were discussed [13].

\section{Data Filtering and Classification of Variants}

After sequencing, raw FASTQ data were obtained and processed via the following steps: (1) Variants were called using the Genome Analysis Toolkit to find sites with differences between the sample sequence data and the reference genome. After alignment of reads against the human reference genome sequence, using standard procedures to identify single nucleotide variants and small insertions and deletions. Variants were annotated using ANNOVAR software and splicing variants were considered up to \pm 10 bp from the exon-intron boundary. (2) Variants were filtered according to quality parameters. (3) Only rare variants in coding sequence or exon-intron boundaries with a frequency under $0.5 \%$ in the Genome Aggregation Database (GnomAD), or variants considered pathogenic in public databases were collected and evaluated for their potential relationship to Mendelian diseases, and were analyzed manually via extensive bibliographic research. (4) Quality of variants was then confirmed. (5) Potential variants were assessed in relation to the index case phenotype and classified following the American College of Medical Genetics and Genomics (ACMG) guidelines [14]. Population (GnomAD) and disease (ClinVar, or Human Gene Mutation Database) databases were used as a starting point to assess the variant's frequency in the gen- 


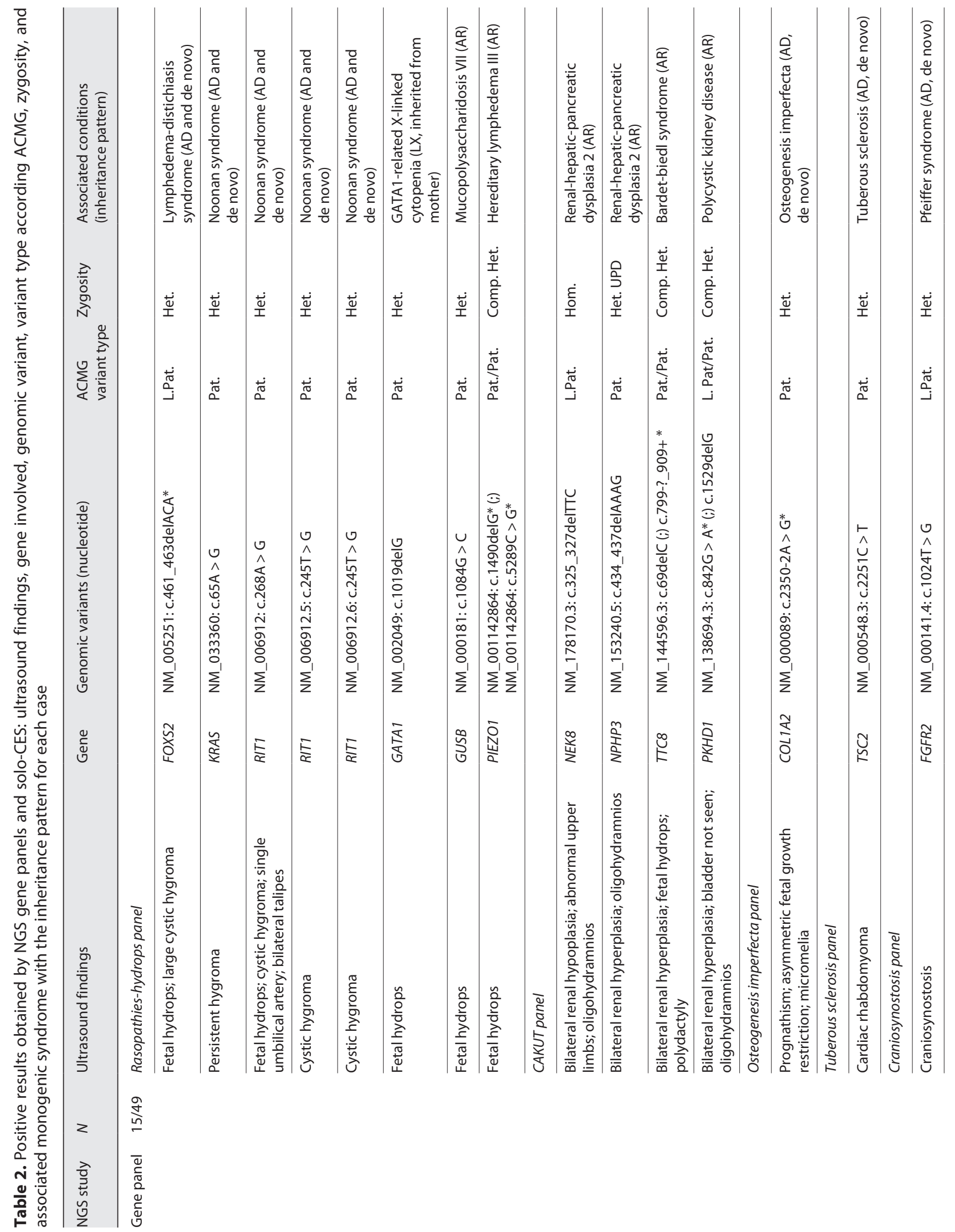




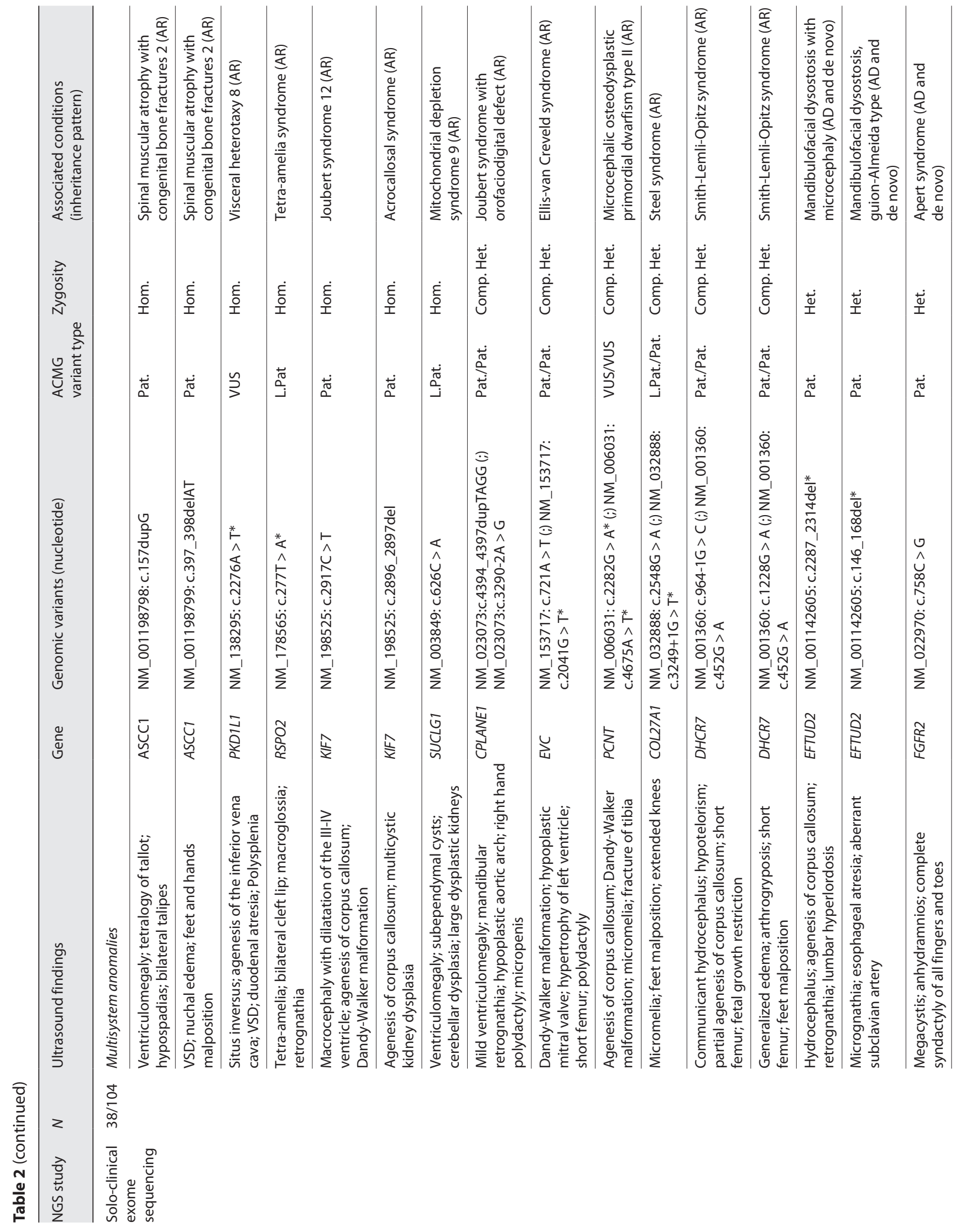




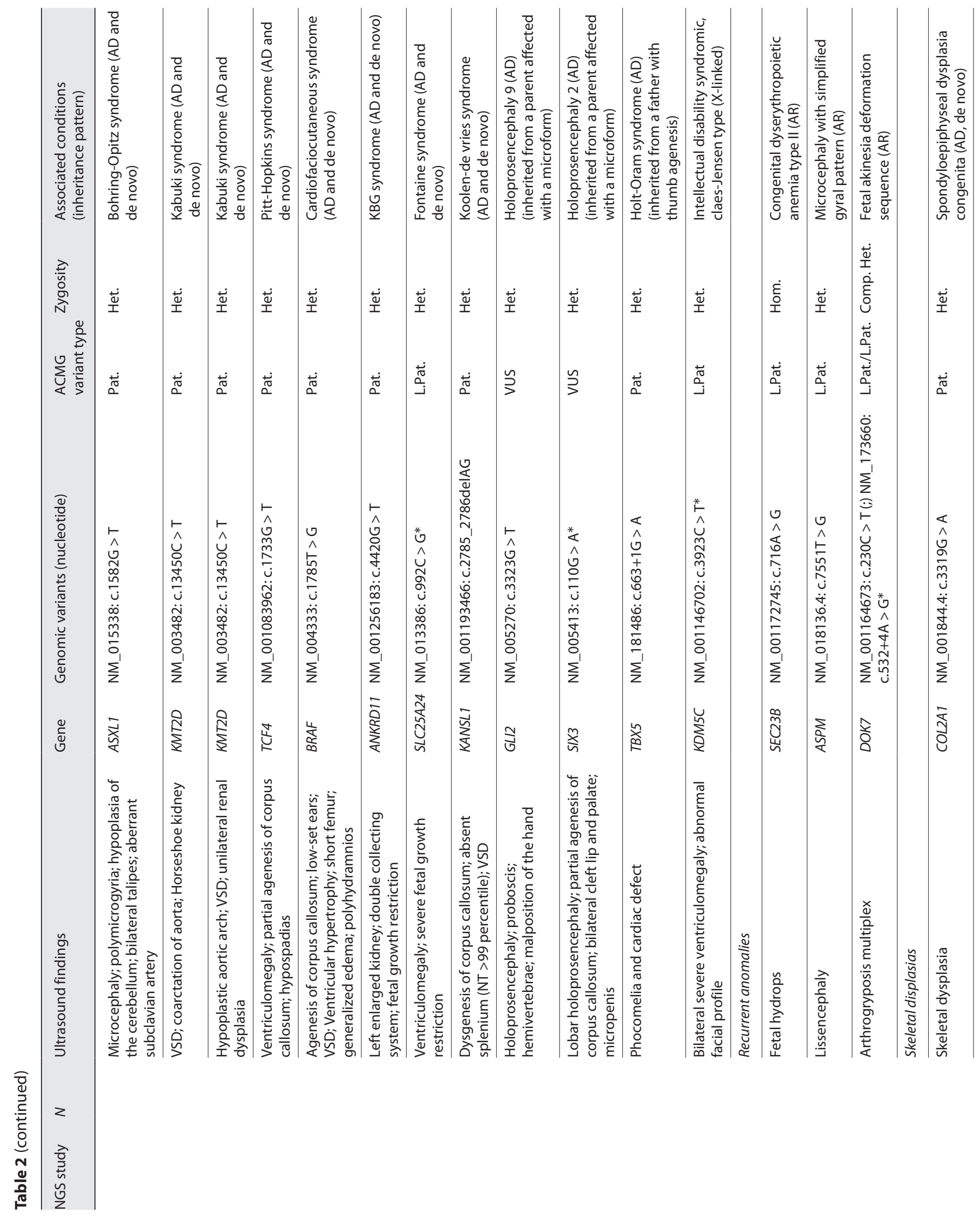


eral population and whether it was already described as pathogenic in disease databases. (6) Furthermore, the Varsome $[15,16]$ algorithm was applied taking into account the type of variant (nonsense, frameshift, exon deletion, and intronic) and computational in-silico predictive programs.

Reports inform about pathogenic, likely pathogenic, and extremely rare variants of uncertain significance (VUS) identified in genes that are related to the index case phenotype or overlapping phenotypes. Secondary findings were reported following the ACMG guidelines for the communication of pathogenic and likely pathogenic variants in actionable genes [17].

Sanger sequencing in the index case and parents was performed to confirm the inheritance pattern of the most promising candidates. Negative results were reviewed to search for possible causative genes and ensure correct coverage in the NGS experiment. A multidisciplinary Clinical Review Committee composed of 6 members: a Maternofetal Medicine specialist and Prenatal Geneticist (A.B.), a Pediatric Geneticist (G.A.), a Perinatal Pathologist (A.N.), a Molecular Geneticist (B.C.), a Genetic Counselor (M.S.), and the Project Coordinator (M.P.), reviewed all the candidate variants and the negative results. The committee could request reviewing the coverage of specific genes in negative results, or expanding the parental segregation analysis or the study of previous siblings or other members of the family.

\section{Ethical Approval}

This study was approved by the Institutional Review Board of the Hospital Clínic de Barcelona (HCB/2018/0077), and written informed consent was obtained from the parents.

\section{Results}

During the 6-year study period (January 2015-October 2020), 149 selected structurally abnormal fetuses with a normal CMA in the absence of a known familial mutation were recruited. All women consented to NGS studies, although one missed the blood draw appointment, resulting in 148 studied fetuses. Mean maternal age was 34 years, range (18-47 years) and the mean gestational age at ultrasound diagnosis was $20+2$ weeks (range 1034 weeks). There were 5 fetuses in which both gene panel and solo-CES were consecutively performed, leading to an overall of 153 NGS studies. In fetuses from our center, 49 gene panels and 69 solo-CES were performed, together with 35 solo-CES in fetuses referred from the other participating centers. The number of cases studied yearly increased from 5 cases (2015), 10 cases (2016), 11 cases (2017), 38 cases (2018), 46 cases (2019), to 43 (2020). In 5 fetuses, after a negative result with the NGS gene panel, the study was extended to solo-CES.

Overall, NGS studies revealed the cause of the anomalies observed in 53 of the 153 fetuses studied, resulting in a 35\% incremental diagnostic yield over CMA (Table 1). According to the ACMG classification, there were 40 
Table 3. Perinatal outcome of the 148 pregnancies studied, according to whether a definitive diagnosis was provided by NGS studies

\begin{tabular}{llllll}
\hline & Stillbirth & $\begin{array}{l}\text { Termination of } \\
\text { pregnancy }\end{array}$ & $\begin{array}{l}\text { Neonatal } \\
\text { survival }\end{array}$ & $\begin{array}{l}\text { Neonatal } \\
\text { death }\end{array}$ & Total \\
\hline Negative result & 7 & 60 & 23 & 5 & 95 \\
Definitive diagnosis provided & 4 & 43 & 4 & 2 & 53 \\
\hline Total & 11 & 103 & 27 & 7 & 148 \\
\hline
\end{tabular}

pathogenic, 22 likely pathogenic, and 7 VUS reassigned by the multidisciplinary Clinical Review Committee as likely causative, after parental segregation studies (online suppl. Table 2 [23-28]). Hence, medical examination of the corresponding parent was indicated when an inherited VUS of a gene with an autosomal dominant inheritance, related to the observed fetal phenotype had an incomplete penetrance or variable expression. Among the 53 monogenic disorders diagnosed, 26 (49\%) had an autosomal dominant inheritance ( 23 caused by a de novo variant), 25 (49\%) had an autosomal recessive inheritance (14 compound heterozygous and 11 homozygous variants), and 2 cases $(3.7 \%)$ had an $\mathrm{X}$-linked recessive inheritance (Table 2).

NGS gene panels were applied in 49 cases, with a $31 \%$ (15/49) diagnostic yield (Table 1). The gene panel most used ( $n=23$ ) was the RASopathies-hydrops gene panel, which in the last years of the study included 130 genes associated with RASopathies and fetal hydrops, and resulted in a 35\% (8/23) diagnostic yield (Table 2). The CAKUT panel was applied in 7 cases of large hyperechogenic kidneys with a 57\% (4/7) diagnostic yield. The OI panel had a $25 \%(1 / 4)$ diagnostic yield (Table 1). Reporting times ranged from 2 to 4 weeks.

Solo-CES demonstrated a 37\% (38/104) diagnostic yield. The observed test performance for fetuses with multisystem major anomalies was 41\% (28/69), 25\% $(3 / 12)$ for recurrent major anomalies, and $50 \%(5 / 10)$ for severe skeletal dysplasias (Table 2). The mean depth of coverage of the targeted coding region was at least $50 \times$. Typically, the turnaround time in negative cases was 3 weeks, while an extra 3 weeks were needed for Sanger sequencing in positive cases.

Repeat testing by solo-CES after a negative gene panel result yielded a diagnosis in 2 of the 5 cases. In the case with a negative result after a lissencephaly panel, a variant was detected in the ASPM gene, not included in the panel (microcephaly with simplified gyral pattern), and in the case of the cardiomyopathy panel, a pathogenic variant in the KMT2D gene (Kabuki syndrome) was detected with solo-CES. A single (1\%) secondary finding was found by

NGS Applied in Structurally Abnormal Fetuses
solo-CES in the MYBPC3 gene, not related to the fetal phenotype (rachischisis and malformed vertebrae and ribs), but causing familial hypertrophic cardiomyopathy. This variant was inherited from the mother who was counseled about this finding and referred to the cardiologist. Regarding incidental findings, a "de novo" pathogenic variant was found in the MED13L gene, associated with intellectual disability, in a case of multisystem anomalies. This gene was included in the filtering of the variants in the CES since it has been described in association with intellectual disability [18].

Regarding pregnancy outcomes, 103 (69\%) of the 148 pregnancies studied were terminated upon parental request, and a definitive diagnosis was reached in $43(42 \%$ diagnostic yield) of these cases, although only 5 (12\%) diagnoses were reported before termination (Table 3 ). Among the 45 continuing pregnancies, a definitive diagnosis was reached in 10 (22\% diagnostic yield) of the cases. In 4 of these, the fetus was delivered stillborn, and among the 6 liveborn fetuses there were 2 neonatal demises. Among the 35 fetuses without a definitive diagnosis from continuing pregnancies, 7 were delivered stillborn, and 28 were born alive, although 5 infants died in the neonatal period and another infant died at 7 months (Table 3).

\section{Discussion}

Our study demonstrates an overall diagnostic yield of $36 \%(53 / 148)$ among fetuses with selected structural anomalies. When the NGS studies managed to provide a causative diagnosis, an accurate prognostic profile for the proband, together with the recurrence risk, were established. The observed diagnostic yield in NGS gene panels applied to those fetal conditions with an available phenotype-driven panel was $31 \%$ (15/49), with the most effective panels identified as the CAKUT and RASopathieshydrops panels. Our approach for exome sequencing using solo-CES, which entails sequencing the fetus alone and interpreting only the OMIM genes, provided a $37 \%$ 
(38/104) diagnostic yield, and was proven particularly efficient in multisystem anomalies, skeletal dysplasias, and recurrent anomalies.

NGS-based diagnostic panels, initially used in pediatrics, have been subsequently applied to prenatal cases when the observed fetal phenotype matched the conditions covered by these gene panels. Gene panels analyze multiple genes at once, ranging from 2 to 140 in our study, selected because they had been described as associated with a specific fetal phenotype. The most characteristic panel is that containing genes for Noonan syndrome, applied in persistent nuchal edema after an increased first trimester nuchal translucency. However, in 2 large prenatal series in which a RASopathies panel was applied, a lower yield (2.9-9.4\%) than that observed in our study $(35 \%)$ was achieved $[19,20]$. This discrepancy may be explained by different inclusion criteria, as we requested not just an isolated increased nuchal translucency (as in the 2 previous studies), but also an increased nuchal fold persisting in the second trimester. The prenatal use of a gene panel for skeletal dysplasias [21] and for CAKUT panel in hyperechogenic large kidneys [10] has also been reported to achieve a remarkable yield. However, in our center, we preferred to use solo-CES for skeletal dysplasias, other than suspected OI, given that the gene coverage of the panel available was found to be incomplete during the first cases of the study. A recent study sought to compare WES with a simulated application of commercial gene panels' diagnostic yield in children susceptible of having a genetically heterogeneous condition, concluding that, had a panel been selected instead, $23 \%$ of WES-diagnosed children would not have been diagnosed [22].

Prenatally, WES can be particularly useful in cases where a clinically available phenotype-driven panel cannot provide an answer, as variants in genes that were not on the specific prenatal panel for the phenotype being tested can be detected by WES [8]. The 2 largest WES studies, carried out in Britain and the United States, were reported in the same issue of the Lancet in February 2019. In the British PAGE study, among 610 fetuses with structural anomalies analyzed by trio-WES, a diagnostic genetic variant was identified in $8.5 \%$ of the fetuses, which recently increased to $11 \%$ in the final analysis (Kilby $\mathrm{M}$. $\mathrm{D}$, personal communication). The American study included DNA samples from 234 eligible trios. Diagnostic genetic variants were identified in $10 \%$ of the families, while variants with bioinformatic signatures, possibly indicative of pathogenicity, but with insufficient evidence to be considered diagnostic, were found in another $20 \%$ of the remaining fetuses [6]. In these 2 large series, the diagnostic yield declined in comparison with previous WES studies and also with our study, due to different inclusion criteria, given that fetal structural anomalies of any kind were included in both studies.

The vast majority of prenatal NGS studies use a trioWES approach, which allows screening 3 exomes simultaneously, from the fetus and the parents. Our group has introduced a simplified exome sequencing method, based on a decreased number of exomes sequenced and genes interpreted. Instead of performing 3 exomes ("trio" analysis), the fetus and both parents, we sequenced the fetus alone ("solo" analysis). Instead of interpreting all the 23,000 genes of the human genome described, we interpreted the 4,000 genes described in association with an abnormal phenotype by the OMIM database, known as the "clinical exome" or "medical exome." In positive cases, Sanger sequencing was subsequently applied for variant confirmation and parental segregation analysis. As expected, the solo-CES cost is less than one third of a trio-WES, and this difference is crucial for any public health system.

The main drawback of the solo-CES approach, as compared with trio analysis, is that a second step is required in positive cases to elucidate whether the candidate variants are inherited from one of the parents or if they are "de novo" findings. We took advantage of the recommended Sanger sequencing confirmation of positive NGS results to simultaneously test the parents. In our study, the turnaround time typically was 6 weeks in positive cases, exceeding by 3 weeks the time for negative cases. This is not an optimal turnaround time if the results would affect pregnancy management. However, in our setting, solo-CES was restricted to pregnancies with severe fetal anomalies, where, often, the decision to terminate the pregnancy had already been made based solely on the ultrasound findings. Otherwise, in our country termination for severe anomalies is allowed until the end of pregnancy. A strong point in favor of sequencing the fetus alone is that we encounter fewer incidental findings in the parental genomes.

Our study is not without limitations. The main limitation is the inability to extrapolate the diagnostic yield for the specific fetal anomalies studied herein to other fetal anomalies not included in this study, as highlighted in the discussion. Proper comparisons between groups are hampered by the resulting small sample size after stratification according to NGS methods. A fair comparison with trio analysis, which would audit whether any pathogenic variants in our cohort were missed, using the solo-CES method, is not possible in the present series, and this should then be considered a limitation and a suggestion for a fur- 
ther study. Another limitation, which is intrinsic to the new technique, is the fact that a large proportion of candidate variants carry an uncertain significance because there is no information about them. Nowadays, exome sequence interpretation is not as straightforward for clinicians as karyotype or CMA. A multidisciplinary team is needed to interpret previously undescribed variants.

The main strength of our study is the clinical applicability of the proposed solo-CES approach, given that results were delivered to the women and could be used clinically for reassessing fetal prognosis and for improving neonatal management in ongoing pregnancies. In addition, this low-cost exome sequencing method is available for less than one-third of the current trio-WES cost, including the cost of parental segregation analysis. Resources applied to health care are limited and a reduced exome sequencing cost may facilitate its introduction in many countries, although a cost-effectiveness analysis is beyond the scope of this study. In contrast, single gene studies appear to be inefficient and gene panels may be useful when the provider regularly updates its gene content.

\section{Ackowledgments}

The authors thank Esther Titos, Gloria Poblador, and M. Dolores Jiménez from the Molecular Core Laboratory of the Medical Diagnostic Center, Hospital Clínic Barcelona; Laia Rodriguez-Revenga from the Genetic and Molecular Department for her help with the fetal DNA samples; and Sandra Oms for her assistance in parental blood draw. The authors would also like to thank Emma Triviño from CatLab, Neus Baena from Parc Taulí Genetic Laboratory, Loreto Martorell from Hospital Sant Joan de Déu, Jordi Rosell from Son Espases Genetic and Molecular Department, and Alberto Plaja from Vall d'Hebron Genetic Laboratory for their help in handling fetal DNA samples. Also, Iosifina Stergiotou for her suggestions regarding the English review of the manuscript.

\section{Statement of Ethics}

This study was approved by Institutional Review Board of Hospital Clinic de Barcelona (HCB/2018/0077), and written informed consent was obtained from parents.

\section{Conflict of Interest}

The authors report no conflict of interest.

\section{Funding Sources}

This study was partly supported by a grant from Fondo de Investigaciones Sanitarias (Grant \#PI17/01153), Instituto de Salud Carlos III, and Fondo Europeo de Desarrollo Regional (FEDER), Ministerio de Economia y Competitividad, Spain. The preliminary results of this study were presented in the top abstracts' session at the 23rd International Conference on Prenatal Diagnosis and Therapy in Singapore (2019).

\section{Author Contributions}

A.T.; S.P.; E.M.; L.M.; E.L.; J.S.; B.M.; E.G.; F.P.; V.B.; O.G.; E.E.; M.L.; and M.C. gave their substantial contributions to the acquisition of data for the work. G.A. and A.N. gave their substantial contribution to the interpretation of the found variants and phenotype. B.C. and M.S carried out the interpretation of exome variants and participated in the committee. They contributed to the manuscript. M.P gave substantial contributions to the design of the work, analysis, and interpretation of data for the work and drafting the work under the supervision of A.B. who revised it critically for important intellectual content and approved the final version to be published, aided in interpreting the results and worked on the manuscript. All authors discussed the results and commented on the manuscript.

\section{Data Availability Statement}

All data generated during this study are included in this article and its online supplementary material files. Further inquiries can be directed to the corresponding author.

\section{References}

NGS Applied in Structurally Abnormal Fetuses
1 Dolk H, Loane M, Garne E. The prevalence of congenital anomalies in Europe. Adv Exp Med Biol. 2010;686:349.

2 Wapner RJ, Martin CL, Levy B, Ballif BC, Eng CM, Zachary JM, et al. Chromosomal microarray versus karyotyping for prenatal diagnosis. N Engl J Med. 2012;367(23):2175-84.

3 Callaway JLA, Shaffer LG, Chitty LS, Rosenfeld JA, Crolla JA. The clinical utility of microarray technologies applied to prenatal cytogenetics in the presence of a normal con- ventional karyotype: a review of the literature. Prenat Diagn. 2013;33(12):1119.

4 Nicolaides KH, Snijders RJM, Campbell S, Gosden CM, Berry C. Ultrasonographically detectable markers of fetal chromosomal abnormalities. Lancet. 1992;340(8821):704.

5 Yang Y, Muzny DM, Reid JG, Bainbridge MN, Willis A, Ward PA, et al. Clinical wholeexome sequencing for the diagnosis of mendelian disorders. N Engl J Med. 2013;369(16): 1502. 
6 Petrovski S, Aggarwal V, Giordano JL, Stosic M, Wou K, Bier L, et al. Whole-exome sequencing in the evaluation of fetal structural anomalies: a prospective cohort study. Lancet. 2019;393(10173):758-67.

7 Lord J, McMullan DJ, Eberhardt RY, Rinck G, Hamilton SJ, Quinlan-Jones E, et al. Prenatal exome sequencing analysis in fetal structural anomalies detected by ultrasonography (PAGE): a cohort study. Lancet. 2019; 393(10173):747-57.

8 Vora NL, Powell B, Brandt A, Strande N, Hardisty E, Gilmore K, et al. Prenatal exome sequencing in anomalous fetuses: new opportunities and challenges. Genet Med. 2017; 19(11):1207.

9 Normand EA, Braxton A, Nassef S, Ward PA, Vetrini $\mathrm{F}, \mathrm{He} \mathrm{W}$, et al. Clinical exome sequencing for fetuses with ultrasound abnormalities and a suspected Mendelian disorder. Genome Med. 2018;10(1):74.

10 Bullich G, Domingo-Gallego A, Vargas I, Ruiz P, Lorente-Grandoso L, Furlano M, et al. A kidney-disease gene panel allows a comprehensive genetic diagnosis of cystic and glomerular inherited kidney diseases. Kidney Int. 2018;94(2):363.

11 Bogliolo M, Pujol R, Aza-Carmona M, Muñoz-Subirana N, Rodriguez-Santiago B, Casado JA, et al. Optimised molecular genetic diagnostics of Fanconi anaemia by whole exome sequencing and functional studies. J Med Genet. 2020;57(4):258.

12 Amberger J, Bocchini CA, Scott AF, Hamosh A. McKusick's Online Mendelian Inheritance in $\mathrm{Man}\left(\mathrm{OMIM}^{\circledR}\right)$. Nucleic Acids Res. 2009; 37(Database issue):D793.

13 Jelin AC, Vora N. Whole exome sequencing: applications in prenatal genetics. Obstet Gynecol Clin North Am. 2018;45(1):69.
14 Richards S, Aziz N, Bale S, Bick D, Das S, Gastier-Foster J, et al. Standards and guidelines for the interpretation of sequence variants: a joint consensus recommendation of the American college of medical genetics and genomics and the association for molecular pathology. Genet Med. 2015;17(5):405.

15 Kopanos C, Tsiolkas V, Kouris A, Chapple CE, Aguilera MA, Meyer R, et al. VarSome: the human genomic variant search engine. Bioinformatics. 2019;35(11):1978.

16 Kopanos C, Tsiolkas V, Kouris A, Chapple C, Aguilera MA, Meyer R, et al. VarSome: the human genomic variant search engine. Bioinformatics. 2019;35(11):1978.

17 Kalia SS, Adelman K, Bale SJ, Chung WK, Eng C, Evans JP, et al. Recommendations for reporting of secondary findings in clinical exome and genome sequencing, 2016 update (ACMG SF v2.0): a policy statement of the American college of medical genetics and genomics. Genet Med. 2017;19(2):249.

18 Adegbola A, Musante L, Callewaert B, Maciel $\mathrm{P}, \mathrm{Hu} \mathrm{H}$, Isidor $\mathrm{B}$, et al. Redefining the MED13L syndrome. Eur J Hum Genet. 2015; 23(10): 1308 .

19 Stuurman KE, Joosten M, Van Der Burgt I, Elting M, Yntema HG, Meijers-Heijboer $\mathrm{H}$, et al. Prenatal ultrasound findings of rasopathies in a cohort of 424 fetuses: update on genetic testing in the NGS era. J Med Genet. 2019;56(10):654.

20 Sinajon P, Chitayat D, Roifman M, Wasim S, Carmona S, Ryan G, et al. Microarray and RASopathy-disorder testing in fetuses with increased nuchal translucency. Ultrasound Obstet Gynecol. 2020;55(3):383.

21 Zhou X, Chandler N, Deng L, Zhou J, Yuan M, Sun L. Prenatal diagnosis of skeletal dysplasias using a targeted skeletal gene panel. Prenat Diagn. 2018;38(9):692.
22 Dillon OJ, Lunke S, Stark Z, Yeung A, Thorne $\mathrm{N}$, Gaff C, et al. Exome sequencing has higher diagnostic yield compared to simulated disease-specific panels in children with suspected monogenic disorders. Eur J Hum Genet. 2018;26(5):644.

23 Vetrini F, D’Alessandro LCA, Akdemir ZC, Braxton A, Azamian MS, Eldomery MK, et al. Bi-allelic mutations in PKD1L1 are associated with laterality defects in humans. Am J Hum Genet. 2016.

24 Le Fevre A, Baptista J, Ellard S, Overton T, Oliver A, Gradhand E, et al. Compound heterozygous Pkd1ll variants in a family with two fetuses affected by heterotaxy and complex Chd. Eur J Med Genet. 2020;63(2): 103657.

25 Rauch A, Thiel CT, Schindler D, Wick U, Crow YJ, Ekici $A B$, et al. Mutations in the pericentrin (PCNT) gene cause primordial dwarfism. Science. 2008;319(80):816.

26 Rodríguez-García ME, Cotrina-Vinagre FJ, Cruz-Rojo J, Garzón-Lorenzo L, CarniceroRodríguez P, Pozo D, et al. A rare male patient with Fontaine progeroid syndrome caused by p.R217H de novo mutation in SLC25A24. Am J Med Genet Part A. 2018.

27 Writzl K, Maver A, Kovačič L, MartinezValero P, Contreras L, Satrustegui J, et al. De novo mutations in SLC25A24 cause a disorder characterized by early aging, bone dysplasia, characteristic face, and early demise. Am J Hum Genet. 2017;101(5):844.

28 Zhang W, Taylor SP, Ennis HA, Forlenza KN, Duran I, Li B, et al. Expanding the genetic architecture and phenotypic spectrum in the skeletal ciliopathies. Hum Mutat. 2018;39(1): 152 